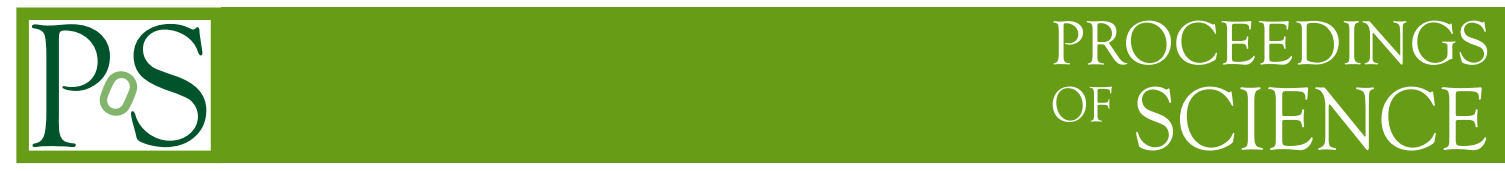

\title{
Neutrino Telescope in Lake Baikal: Present and Future
}

A.D. Avrorin ${ }^{a}$, A.V. Avrorin ${ }^{a}$, V.M. Aynutdinov ${ }^{a}$, R. Bannash ${ }^{g}$, I.A Belolaptikov ${ }^{b}$, V.B. Brudanin ${ }^{b}$, N.M. Budnev ${ }^{c}$, G.V. Domogatsky ${ }^{a}$, A.A. Doroshenko ${ }^{a}$, R. Dvornickýy ${ }^{b, h}$, A.N. Dyachok ${ }^{c}$, Zh.-A.M. Dzhilkibaev ${ }^{a}$, L. Fajt ${ }^{h, i}$, S.V Fialkovsky ${ }^{e}$, A.R. Gafarov ${ }^{c}$, K.V. Golubkov ${ }^{a}$, N.S. Gorshkov ${ }^{b}$, T.I. Gress ${ }^{c}$, R. Ivanov ${ }^{b}$, K.G. Kebkal ${ }^{g}$, O.G. Kebkal ${ }^{g}$, E.V. Khramov ${ }^{b}$, M.M. Kolbin ${ }^{b}$, K.V. Konischev ${ }^{b}$, A.V. Korobchenko ${ }^{b}$, A.P. Koshechkin ${ }^{a}$, A.V. Kozhin ${ }^{d}$, M.V. Kruglov ${ }^{b}$, M.K. Kryukov ${ }^{a}$, V.F. Kulepov ${ }^{e}$, M.B. Milenin ${ }^{a}$, R.A. Mirgazov ${ }^{c}$, V. Nazari ${ }^{b}$, A.I. Panfilov $^{a}$,

D.P. Petukhov ${ }^{a}$ E.N. Pliskovsky ${ }^{b}$, M.I. Rozanov ${ }^{f}$, E.V. Rjabov ${ }^{c}$, V.D. Rushay ${ }^{b}$, G.B. Safronov ${ }^{b}$, B.A. Shaybonov ${ }^{b}$, M.D. Shelepov ${ }^{a}$, F. Šimkovic ${ }^{*, b, h, i}$, A.V. Skurikhin ${ }^{d}$, A.G. Solovjev ${ }^{b}$, M.N. Sorokovikov ${ }^{b}$, I. Štekl $^{i}$, O.V. Suvorova ${ }^{a}$, E.O. Sushenok ${ }^{b}$, V.A. Tabolenko ${ }^{c}$, B.A. Tarashansky ${ }^{c}$, S.A. Yakovlev ${ }^{g}$

${ }^{a}$ Institute for Nuclear Research, Russian Academy of Sciences, Moscow, 117312 Russia

${ }^{b}$ Joint Institute for Nuclear Research, Dubna, 141980 Russia

${ }^{c}$ Irkutsk State University, Irkutsk, 664003 Russia

${ }^{d}$ Institute of Nuclear Physics, Moscow State University, Moscow, 119991 Russia

e Nizhni Novgorod State Technical University, Nizhni Novgorod, 603950 Russia

${ }^{f}$ St. Petersburg State Marine Technical University, St. Petersburg, 190008 Russia

${ }^{g}$ EvoLogics Gmbh, Germany

${ }^{h}$ Comenius University, Mlynska Dolina F1, Bratislava, 84248 Slovakia

${ }^{i}$ Czech Technical University in Prague, Prague, 12800 Czech Republic

E-mail: simkovicefmph.uniba.sk

A significant progress in the construction and operation of the Baikal Gigaton Volume Detector in Lake Baikal, the largest and deepest freshwater lake in the world, is reported. The effective volume of the detector for neutrino initiated cascades of relativistic particles with energy above 100 $\mathrm{TeV}$ has been increased up to about $0.25 \mathrm{~km}^{3}$. This unique scientific facility, the largest operating neutrino telescope in Northern Hemisphere, allows already to register two to three events per year from astrophysical neutrinos with energies exceeding $100 \mathrm{TeV}$. Preliminary results obtained with data recorded in 2016-2018 are announced. Multimessenger approach is used to relate finding of cosmic neutrinos with those of classical astronomers, with X-ray or gamma-ray observations and the gravitational wave events.

36th International Cosmic Ray Conference -ICRC2019-

July 24th - August 1st, 2019

Madison, WI, U.S.A.

${ }^{*}$ Speaker. 


\section{Introduction}

High-energy neutrino astronomy, a very recent and lively research field, has emerged by a construction of gigaton volume neutrino telescopes deep in ice and under water at both the Southern (IceCube) and Northern (Baikal-GVD, KM3NeT) Hemispheres. Its primary goal is to study the most distant, powerful energy sources in the universe using high-energy neutrinos. The subject of interest are astrophysical sources, namely the collision and merger of a pair of neutron stars, or a neutron star with a black hole, active galactic nuclei powered by mass accretion onto black hole at the center of its host galaxy, etc. They can be observed by a detection of astrophysical neutrinos in the TeV-PeV range coming from outside our galaxy. The high energy universe is a great laboratory to search also for fundamental properties of matter, in particular dark matter particles, axions, magnetic monopoles, new light bosons etc.

Recently, IceCube telescope confirmed that blazars, an intense source of radiation powered by a supermassive black hole, produce ultra-high energy neutrinos [1]. A $290 \mathrm{TeV}$ neutrino was registered that could be traced back to TXS 0506+056 blazar that emits copious amounts of gamma rays. In this way the era of multimessenger astronomy has started allowing to look at sky simultaneously with light, neutrinos, and gravitational waves.

In this contribution a significant progress in construction and performance of underwater neutrino telescope in Lake Baikal is reviewed. A description of the Baikal-GVD telescope working principles as well as the reconstruction of high-energy events which are identified above the background of atmospheric neutrinos are shortly presented [2].

\section{The Baikal-GVD neutrino telescope}

Next generation cubic kilometer scale neutrino telescope Baikal-GVD is currently under construction in Lake Baikal since 2015. The detector is specially designed for search for high energies neutrinos whose sources are not yet reliably identified.

The Baikal-GVD detector is 3 dimensional lattice of optical modules (OMs) that house photomultiplier tubes to detect Cherenkov light from secondary muons and cascades produced in neutrino interactions in the water of the lake. The OMs are arranged on vertical load-carrying cables to form strings. The configuration of telescope consists of functionally independent clusters of strings, which are connected to shore by individual electro-optical cables. Each cluster comprises in eight strings 288 optical modules arranged at depths down to $1300 \mathrm{~m}$. Seven peripheral strings are uniformly located at a $60 \mathrm{~m}$ distance around a central one. OMs on each string are combined in sections, detection units of telescope. The distances between the central strings of neighboring clusters are about $300 \mathrm{~m}$.

A basic element of operation of the Baikal-GVD detector is a section of 12 OMs distributed vertically along the string, spaced by $15 \mathrm{~m}$, and a central electronics module $(\mathrm{CeM})$. There are 3 sections of 36 OMs per string and 8 strings in cluster, each is an independently working subarray of the Baikal-GVD. As light sensor of OM a single photomultiplier tube (PMT) Hamamatsu R7081-100 with a 10-inch hemispherical photocathode and quantum efficiency up to $35 \%$ is exploited [3]. The PMT signals are amplified and transmitted to the ADC units to measure the pulse shape with a sampling rate $200 \mathrm{MHz}$ [4]. Each ADC unit is equipped with quartz oscillators that 

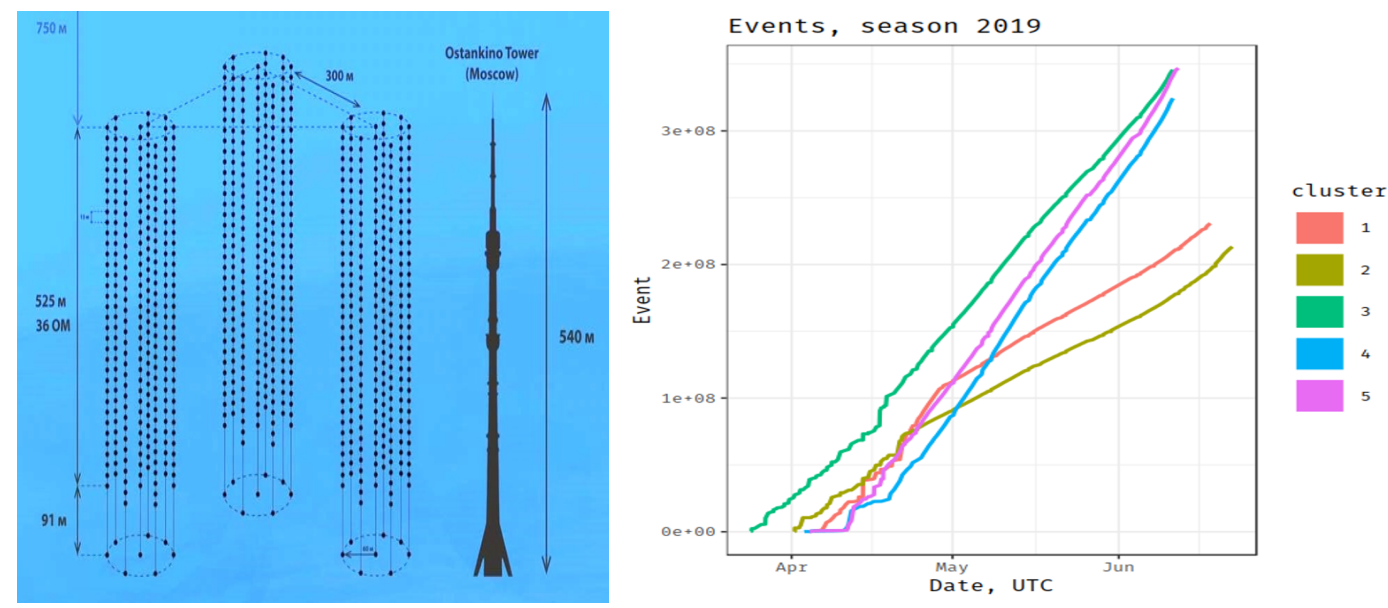

Figure 1: Schematic drawing of the Baikal-GVD three clusters operated in 2018 (left panel) and integrated number of events recorded by the clusters from April to June 2019 (right panel).

allow measuring the arrival time of photons. A global trigger signal is used to unify the event times measured by different ADC units within the same cluster to a single time scale with accuracy $\sim 2$ ns. The trigger condition is a coincidence of pulses from any neighboring optical modules with thresholds $\sim 2$ and $\sim 5$ photoelectrons. Once trigger condition is fulfilled, a request signal is generated to the cluster center module (CC) through $1 \mathrm{~km}$ long line. CC generates an acknowledgement signal to all CMs in a cluster, when the request signal is received. When CM is caught the signal, a timestamp is defined and the CM starts to form data. The programmable gate array memory buffer allows to acquire the OM signal waveform for a time interval as long as $5 \mu \mathrm{s}$, this information then is transferred to the shore station and stored as raw data in binary files. Such a trigger system approach allows to have all signal waveforms on each channel in an event from only one triggered pair of adjacent channels, while the data arriving from sections can be processed in real time mode. All raw data, for an amount as large as 40 Gbyte per cluster per day, are transferred to the shore station, stored there, and then transmitted at $5 \mathrm{Mbit} / \mathrm{s}$ on a $40 \mathrm{~km}$ channel to Baikalsk (Irkutsk region), where data are archived and transferred through a high-bandwidth internet to the central storage and processing facility in the JINR in Dubna (Moscow region) every 4 hours.

Pulse shape analysis allows determining the number of signals coming from the PMT, their charges and registration times. On the basis of measurements of the time of registration of the pulses and their charges on all triggered channels, the main parameters of an event are determined: the direction and energy of muons and cascades, respectively. The accuracy of the direction reconstruction of muon tracks amounts to $0.5^{\circ}$, and that of the energy of cascades to about $20 \%$. For amplitude and time calibration of the detector, LED calibration sources are used. They are located both in the optical modules and in separate deep-water glass spheres (LED matrix). Each OM comprises two LEDs, the light from which spreads up along the string. These LEDs are employed for channel calibration within sections of OMs. The LED matrices are used for inter-section time calibration. Each matrix comprises 12 synchronized LEDs flashers, pulses from which can be detected by several sections. The accuracy of the time calibration is about $2 \mathrm{~ns}$ [5]. Laser sources are used for inter-cluster calibration. The laser calibration source generates light flashes with about $10^{15}$ 
photons at maximum power running. The laser wavelength is $532 \mathrm{~nm}$, the pulse duration about $1 \mathrm{~ns}$. The laser is located approximately in the center of the group of three clusters, at the level of the middle of the lower sections of the optical modules (see Figure 1, left panel). The laser is equipped with a diffuser that forms a quasi-isotropic light flux. One laser source allows calibrating three clusters simultaneously. Details of data acquisition, basic controls, methods of calibrations, hard-and soft-ware triggers can be found in [4] and [6].

The first phase (Phase 1) of the construction of the Baikal-GVD telescope with 8 clusters with 2304 light sensors in total covering $0.4 \mathrm{~km}^{3}$ of instrumented volume is expected to be finished in 2021. However, starting April 2015, when the first GVD cluster Dubna was commissioned, data from the first clusters of the detector are already collected, analyzed and exploited for a development of data analysis software. Since April 2018 the telescope had been successfully operated in complex of three functionally independent clusters i.e. sub-arrays of optical modules (OMs) hosting 864 OMs on 24 vertical strings. A significant amount of work has been accomplished during this year winter expedition resulting in operation of two new clusters, altogether 5 clusters with effective volume of $0.25 \mathrm{~km}^{3}$, starting April 2019. In Fig. 1 (right panel) the event accumulation rate of the five clusters from April to June 2019 is presented. The change in slope for clusters 1 and 2 is due to raising the trigger threshold. Their DAQ (already optimized for the last 3 clusters) was challenged by the high data rates. About $1.5 \times 10^{9}$ events have been recorded, with a data taking efficiency of $90 \%$ for single clusters and almost $100 \%$ with at least one cluster taking data. The current effective volume of the Baikal-GVD detector is just one fourth of the size of the present world leader - the IceCube Neutrino Observatory at the South Pole. A possible extension of Phase 1 to Phase 2 with construction of additional 14 clusters will depend on the performance and physical output of the Baikal GVD detector in 2021.

\section{On the search for high energy neutrino events and their origin}

To priority tasks of the Baikal-GVD telescope belong a search for astrophysical neutrino sources and neutrino events correlated in time and direction with sources of variable luminosity what can be achieved by a study of tracks ascribed to $v_{\mu}, \bar{v}_{\mu}$ and high-energy cascade events ascribed to $v_{e}, \bar{v}_{e}$ and $v_{\tau}, \bar{v}_{\tau}$. The reconstruction of Cherenkov radiation being emitted by cascade particles and straight-line moving muons generated in the high energy neutrino interactions with Baikal water allows to conclude about energies and direction of incoming neutrinos. The BaikalGVD telescope has modular structure what allows to collect and analyze data since April 2015, when the first GVD cluster was commissioned and the neutrino telescope has been in a permanent operation.

\subsection{Detection of muon neutrinos}

A search for upward moving muon neutrinos having a collision with water environment of the Baikal-GVD detector was performed with data collected by the first cluster of the telescope in 2016. For analysis based on precise measurements of optical module positions, timing and amplitude calibrations a set of 70 runs in which detector demonstrated stable behavior was chosen. The total exposition time of this sample of data is close to 33 days. 

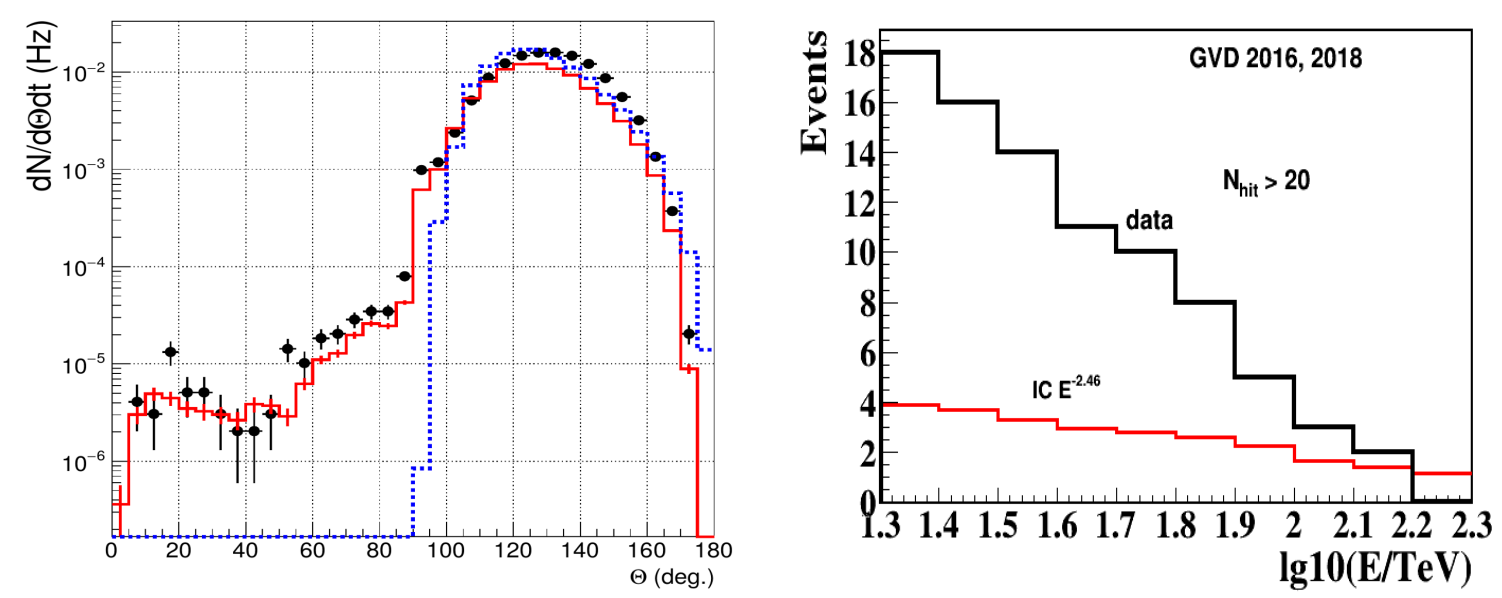

Figure 2: Left panel: Zenith angle distribution reconstructed with data sample of atmospheric muon-like events (black dots) and determined with Monte Carlo simulation of muons (red line) and upward moving muon neutrinos (blue line). Right panel: Cumulative energy distribution of experimental events (black histogram) end events expected from astrophysical flux with $E^{-2.46}$ energy spectrum and IceCube normalization (red histogram).

The collection of PMT signals in each event includes noise pulses due to PMT dark current and to chemiluminescence background in the Baikal water. Rates of noise impulses vary between 20 and $100 \mathrm{kHz}$ depending on season and depth. The noise pulses are rejected with help of the causality requirement: $\left|t_{i}-t_{j}\right| \leq \frac{\Delta R_{i j}}{c_{w}}+t_{s}$, where $t_{i}, t_{j}$ are impulse times, $\Delta R_{i j}$ is distance between modules, $c_{w}$ is speed of light in water and $t_{s}=10 \mathrm{~ns}$. A simple track position and direction estimation is done for each causally-connected group. OM hits which do not obey model of muon propagation and direct Cherenkov light emission are excluded in a gradually tightening set of cuts on hit residuals. The set of hits selected with this procedure has a noise contamination at the level of 1-2\% depending on the elevation of the muon track. Selected set of pulses is used for the track fit under the assumption of direct Cherenkov emission from the muon track. The resulting median resolution of the procedure as measured in the up-going neutrino Monte Carlo sample is at the level of 1 degree.

In Fig. 2 (left panel) the reconstructed angular distribution of muon-like events deduced from the data sample is compared with Monte Carlo (MC) simulation of the detector response to atmospheric muons and upward propagating muon neutrinos. Good agreement in shape and rate between both muon distributions is achieved.

A procedure based on a boosted decision tree (BDT) as implemented in the TMVA framework [7] was developed for the selection of neutrino events. A set of quality variables was reconstructed for each event and used for the BDT discriminant. The BDT was trained on events reconstructed as upward going in MC samples of atmospheric upward going neutrinos (signal) and atmospheric muons (background). Then BDT value was reconstructed for the data events. Those events with BDT value larger than found value for good discrimination of signal and background were selected. Overall, 23 neutrino candidate events for 33 live days were found in data sample, while 42 events are expected from up-going neutrino MC. Number of expected background from atmo- 

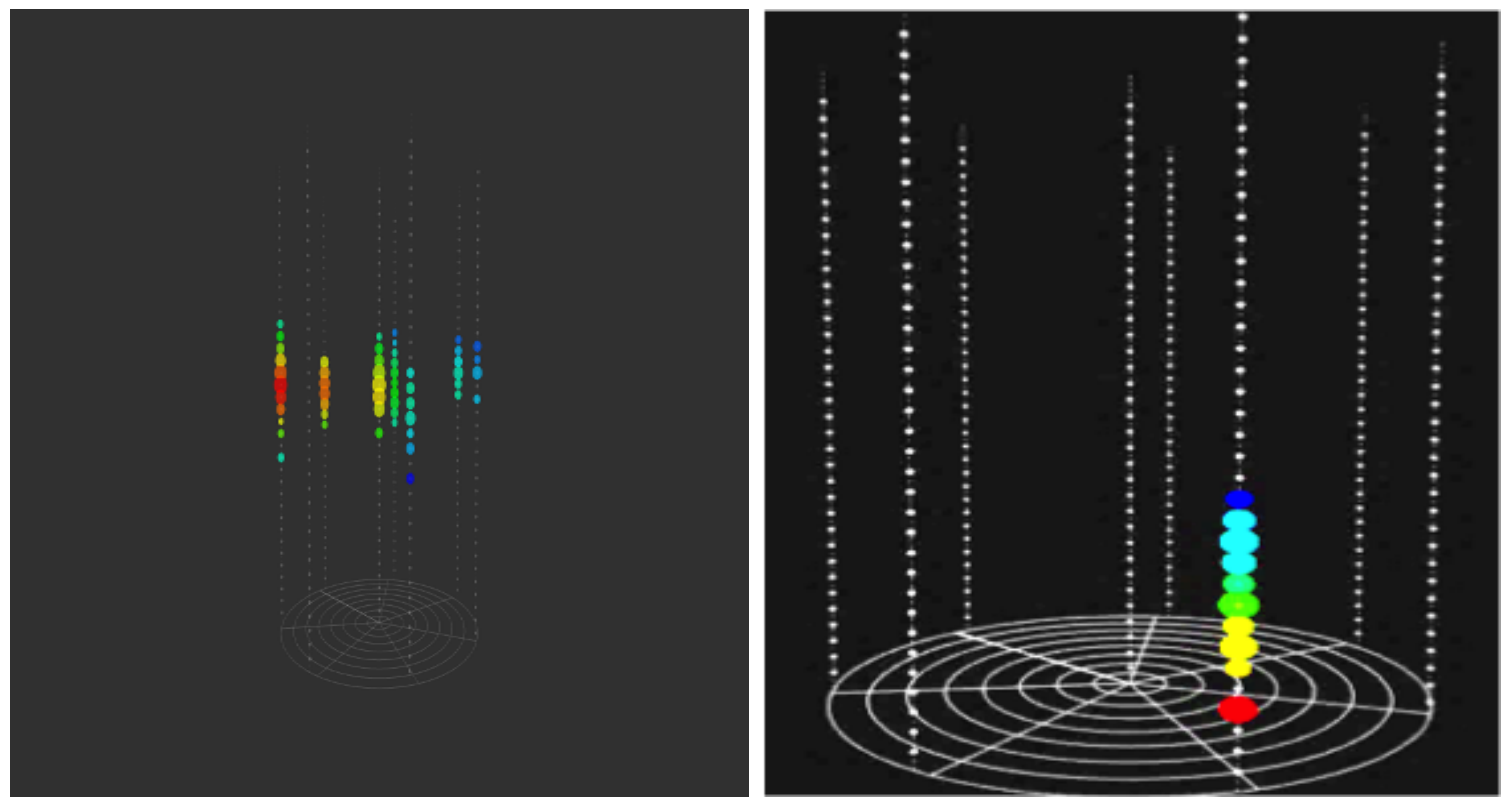

Figure 3: Left panel: Cascade recorded on April 29, 2016 (see text). Right panel: Reconstructed vertical event with 10 hit OMs.

spheric muons is about 6. Improvements in quality of track-like reconstruction is the next iteration of the data sample analysis.

\subsection{Cascade detection by Baikal-GVD detector}

Currently, we do not have a clear theoretical idea about the sources of cosmic neutrinos and how intense they are. We can categorize them as point and sporadic sources which might be related to high-energy processes in astrophysical objects and $\gamma$-ray bursts, respectively. If many extragalactic sources contribute to an intense and measurable neutrino emission we end up with a diffuse flux of cosmic neutrinos. It can be related also to the interaction between cosmic rays and Galactic gas/radiation fields resulting in the flux with $E^{-2.19}$ spectrum for energy above $100 \mathrm{TeV}$. All these sources of cosmic neutrinos can be investigated with neutrino telescopes with a volume about one kilometer cube or slightly smaller. A necessary condition for it is that background processes in the neutrino detector are well understood.

Cosmic ray interactions in the atmosphere produce neutrinos at high energy. These constitute a diffuse, continuous and everywhere present flux, consisting mostly of $v_{\mu}, \bar{v}_{\mu}$ with a spectrum distributed as $E_{v}^{-3.7}$ in the region $100 \mathrm{GeV}-100 \mathrm{TeV}$ with some uncertainty especially at high energies. Together with muons coming from above, these neutrinos constitute the irreducible background when searching for cosmic neutrinos.

The exciting discovery of a diffuse flux of $\mathrm{TeV}$ to $\mathrm{PeV}$ neutrinos of undoubted astrophysical origin was achieved with the cubic kilometer IceCube neutrino detector in 2013. The obtained results demonstrate the importance of the cascade mode of neutrino detection with neutrino telescopes. The Baikal collaboration has long-time experience in the search for diffuse neutrino flux with NT200 telescope by using of cascade mode $[8,9]$. Baikal-GVD telescope has the potential 
to record astrophysical neutrinos with flux values measured by IceCube even at early phases of construction by selecting cascade events generated by neutrino interactions in the sensitive volume of the detector.

To search for high-energy neutrinos of astrophysical origin the data collected by one cluster in 2016 and by three clusters in 2018 have been used. A data sample of $3.8 \times 10^{9}$ events has been accumulated by the array trigger, which corresponds to 872 one cluster live days. After applying procedures of cascade vertex and energy reconstruction for hits with charge higher than 1.5 ph.el., 417 cascade-like events with OMs hit multiplicity $N_{h i t}>13$ have been selected. The requirement of high hit multiplicity allows substantial suppression of background events from atmospheric muon bundles. 18 of selected events have $N_{h i t}>20$ and 3 of them where reconstructed with energies above $100 \mathrm{TeV}$ and satisfy the requirements for astrophysical neutrino selection. The calculations of the probability to obtain such high multiplicity events from atmospheric muons and neutrinos are in progress. One of these three events which was recorded on 29.04. 2016 is shown in Fig. 3 (left panel). Each sphere represents an OM. Colors represent the arrival times of the photons where red indicates early and blue late times. The size of spheres is a measure for the recorded number of photo-electrons. Cumulative energy distribution of 18 experimental events with $N_{\text {hit }}>20$ (black histogram) and distribution of events expected from neutrino flux of astrophysical origin with power low energy spectrum and IceCube normalization $1.7 \times 10^{-10} E^{-2.46}$ $\mathrm{TeV}^{-1} \mathrm{~cm}^{-2} \mathrm{~s}^{-1} \mathrm{sr}^{-1}$ (red histogram) are shown in Fig. 2 (right panel). For energies above $100 \mathrm{TeV}$ 1.44 events are expected from IC flux. In energy range below $100 \mathrm{TeV}$ the data are dominated by background events from atmospheric muons. For energies above $100 \mathrm{TeV}$ higher statistics is required for observation of the astrophysical neutrino flux.

Separately, a data analysis approach was developed for nearly vertical upward going muons, that takes into account particular features of single string event reconstruction. Within the whole data sample of 182 live days in 2016 there have been selected 5674 neutrino candidates. In this sample, there was one candidate event with 10 hits, 6 events with 8 hits, 15 events with 7 hits and 144 events with 6 hits. No candidate events with 9 hits were found. The one event with 10 hits is shown in Fig. 3 (right panel). Expected near vertical neutrino fluence can exceed number of background atmospheric neutrino events as long as there can be an additional flux arising from the Earth's core. For the hypothesis of dark matter in form of weakly interacting massive particles, WIMPs have been accumulated inside the Earth during a time comparable to the universe age and currently weakly annihilate into ordinary matter, producing high energy pairs of neutrinos and antineutrinos through decays of particles.

\subsection{Search for high-energy neutrinos associated with GW170817}

The most interesting source of variable luminosity of multimessenger interest was the galaxy NGC 4993, where binary neutron stars merged and produced a gravitational wave GW170817, that was registered by the Advanced LIGO and Advanced Virgo observatories [10]. A short GRB (GRB170817A), associated with GW170817, was detected by Fermi-GBM and INTEGRAL. Optical observations allowed the precise localization of the merger in the galaxy NGC 4993 at a distance of $\sim 40 \mathrm{Mpc}$. High-energy neutrino signals associated with the merger were searched for by the ANTARES and IceCube neutrino telescopes in muon and cascade modes and the Pierre Auger Observatory [11] and Super-Kamiokande [12]. Two different time windows were used for 

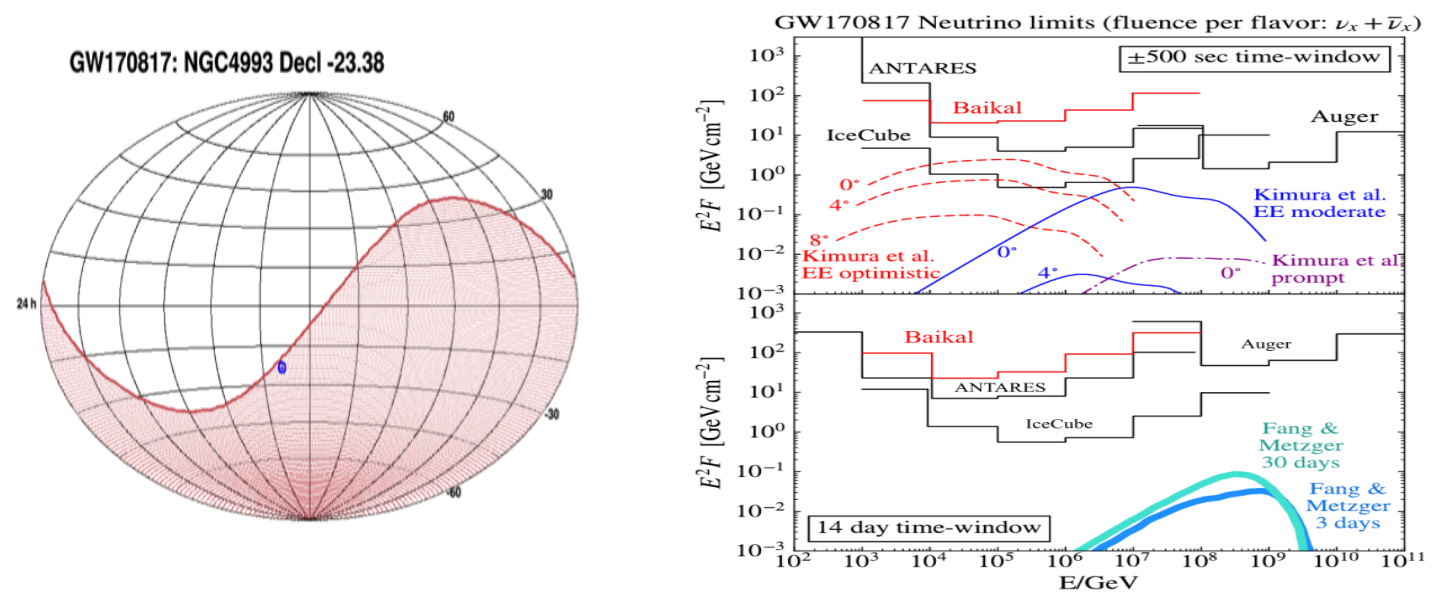

Figure 4: Left panel: Horizon of the Baikal-GVD in alert time of the GW170817A (blue point). Right panel: The Baikal-GVD upper limits on neutrino fluences from direction of the GW170817A (see text).

the searches. First, a $\pm 500 \mathrm{~s}$ time window around the merger was used to search for neutrinos associated with prompt and extended gamma-ray emission [13, 14]. Second, a 14-day time window following the GW detection, to cover predictions of longer-lived emission processes $[15,16]$. No significant neutrino signal was observed by the neutrino telescopes.

Two Baikal GVD-clusters were in operation in 2017. Off-line analysis has been done in cascade mode to search for neutrino signal associated with GW170817A. In looking for fluence in prompt emission $( \pm 500$ seconds) from GW170817A the source was located slightly below the horizon for Baikal-GVD (zenith angle $93^{\circ}$, horizon is shown in Fig. 4 (left panel)). No neutrino events associated with this source have been found in cascade search mode both in the prompt emissions and in the emission delayed on 14 days after alert times. More details of analysis are presented in Ref. [17]. Assuming $E^{-2}$ spectral behavior and equal fluence in neutrino flavors, upper limits at $90 \%$ c.l. have been derived on the neutrino fluence from GW170817A for each energy decade and are shown in Fig. 4 (right panel).

\section{Conclusion}

An important progress has been achieved in the construction of the Baikal-GVD telescope by putting in operation 5 clusters with effective volume of one fourth of the $\mathrm{km}^{3}$. To reach an optimal performance of the Baikal-GVD detector advanced calibrations and analysis techniques have to be further developed, tested and implemented in data processing. Currently, the identification of high energy neutrinos is performed via muon tracks and cascade events in the detector and can be extended also to detection of double pulses later. By exploiting collected data sample of 2015-2018 years the first high-energy cascade events were determined, which might be due to astrophysical high energy neutrinos. In addition, the first neutrino-like events were reconstructed in muon track mode. The attention was concentrated also on the search for neutrinos associated with GW170817A. It was concluded that no high energy neutrino events from desired direction were registered by two Baikal-GVD clusters in operation and an upper limits on the neutrino flu- 
ences were derived. With increasing data records of steadily extending Baikal-GVD detector the importance of the largest fresh water neutrino telescope for multimessenger study of astrophysical objects is growing. There are no doubts that Baikal-GVD telescope will offer a unique view on our universe and provide powerful insights into the performance of some of the most energetic and enigmatic objects in the cosmos.

\section{Acknowledgements}

This work was supported by the Russian Foundation for Basic Research (Grants 16-29-13032, 17-0201237).

\section{References}

[1] M.G. Aartsen et al., IceCube Coll., Science 361, 147 (2018).

[2] A.D. Avrorin et al., Baikal-GVD Coll., EPJ Web Conf. 209, 01015 (2019).

[3] A.D. Avrorin et al., Baikal-GVD Coll., EPJ Web Conf. 116, 01003 (2016).

[4] A.D. Avrorin et al., Baikal-GVD Coll., Instr. Exper. Tech. 57, 262 (2014).

[5] A.D. Avrorin et al., Baikal-GVD Coll., EPJ Web Conf. 207, 07003 (2019).

[6] A.D. Avrorin et.al., Baikal-GVD Coll., PoS ICRC2017, 1046 (2017).

[7] A. Hoecker et al., arXiv:physics/0703039 [physics.data-an], 2007.

[8] V. Aynutdinov et al., Baikal Coll., Astropart. Phys. 25, 140 (2006).

[9] A.D. Avrorin et al., Astronomy Letters 35, 651 (2009).

[10] B. Abbott et al., Phys. Rev. Lett. 119, 161101 (2017).

[11] A. Albert et al., Astrophys. J. 850, L35 (2017).

[12] K. Abe, B., et al., Super-Kamiokande Coll., Astrophys. J. 857, L4 (2018).

[13] B. Baret et al., Astropart. Phys. 35, 1 (2011).

[14] S. Kimura et al., Astrophys. J. 848, L4 (2017).

[15] H. Gao et al., , Phys. Rev. D 88, 043010 (2013).

[16] K. Fang and B. Metzger, Astrophys. J. 849, 153 (2017).

[17] A.D. Avrorin et al., Baikal-GVD Coll., JETP Lett. 108, 787 (2018). 